\title{
PREVALÊNCIA DE LESÕES ENDOMETRIAIS EM MULHERES OBESAS ASSINTOMÁTICAS
}

Daniela Alves da Cruz Gouveia*, luis Bahamondes, José Mendes Aldrighi, Sonia Tamanaha, Alessandra Lorenti Ribeiro, Tsutomu Aoki

Trabalho realizado na Faculdade de Ciências Médicas da Santa Casa de São Paulo

\author{
*Correspondência \\ Rua Dom Paulo Pedrosa, 971 \\ apto 62 \\ Real Parque - São Paulo/ SP \\ Cep: 05687-002 \\ Tel. /Fax: (11) 3759-1633 \\ daniacg@uol.com.br
}

\begin{abstract}
RESUMO
Oвjetivos. A obesidade representa importante fator de risco para alterações endometriais. O presente estudo teve por objetivo avaliar a prevalência de lesões endometriais, como pólipos, hiperplasia e câncer de endométrio em mulheres obesas assintomáticas, assim como reconhecer os fatores de risco associados.

Métodos. Entre dezembro de 2004 e fevereiro de 2006, em estudo transversal foram avaliadas 94 mulheres obesas (índice de massa corpóreo $\geq 30 \mathrm{Kg} / \mathrm{m}^{2}$ ), divididas em dois grupos com 47 participantes cada: em idade reprodutiva e após a menopausa. Foram analisados: história clínica, exame físico (antropométrico/ginecológico), determinações bioquímicas e avaliação do endométrio por ultra-sonografia endovaginal, biópsia e histeroscopia (para confirmar patologia endometrial).

Resultados. Nas mulheres em idade reprodutiva foram encontrados 12,8\% de casos de patologia endometrial, que se associaram significativamente com as elevações da idade, hipertensão arterial (HAS), colesterol e LDL-c. Após a menopausa, foram identificadas 40,4\% de lesões endometriais que se associaram significativamente com pressão arterial sistólica (PAS) $\geq 140 \mathrm{mmHg}$, elevaçãa do LDL-c e da estrona. Dois casos de câncer de endométrio foram constatados, sendo um em cada grupo.

Conclusão. $O$ aumento de incidência da obesidade nos últimos anos tem elevado os fatores de risco para o câncer de endométrio. Na idade reprodutiva tivemos um pequeno tamanho amostral de alterações endometriais; com isso, poderíamos apenas sugerir, uma maior atenção àquelas com idade superior a 40 anos, que apresentem HAS e/ou elevação do LDL-C. O status menopausal eleva o risco de lesão endometrial; associado com elevações da PAS, LDL-c e/ou estrona, elas se tornarão candidatas à biópsia de endométrio, visando o diagnóstico precoce do câncer, decisivo para o prognóstico favorável da mulher.
\end{abstract}

Unitermos: Obesidade. Endométrio. Pré-menopausa. Pós-menopausa. Hipertensão. Colesterol.

\section{INTRODUÇÃO}

A obesidade é uma doença grave, multifacetada e de genética complexa, associada à elevada morbimortalidade!. A influência dos fatores ambientais decorre do desequilíbrio energético entre sedentarismo e excesso de consumo de alimentos hipercalóricos, levando a um aumento nas taxas de mortalidade?

Atualmente, a obesidade representa um problema de saúde pública mundial; sua prevalência na população feminina mostra incremento em todas as idades acentuando-se durante os anos da transição para a pós-menopausa ${ }^{3}$. A Organização Mundial da Saúde ${ }^{4}$ estima que haja 300 milhões de indivíduos obesos e um bilhão de pré-obesos no mundo, sendo que no Brasil este número está em expressivo crescimento ${ }^{5-6}$.

Estudos populacionais mostram que, além do aumento do índice de massa corpórea $\left(\mathrm{IMC} ; \mathrm{Kg} / \mathrm{m}^{2}\right)$ se associar a um maior risco de agravos à saúde e mortalidade em ambos os sexos, principalmente para doença cardiovascular $(\mathrm{DCV})^{7}$, nas mulheres, em particular, a obesidade isoladamente se responsabiliza por um incremento de três a dez vezes no risco do câncer de endométrio.

A incidência mundial do câncer de endométrio está em franca ascensão; de fato, estudos epidemiológicos desvelam que mais de $40 \%$ dos casos são atribuídos ao excesso de peso ${ }^{\circ}$, acometendo $80 \%$ das mulheres no estágio da pós-menopausa, na idade entre 50 e 60 anos $^{10}$. A prevalência do câncer endometrial, em mulheres com obesidade abdominal, mostra-se aumentada nas portadoras de hipertensão arterial sistêmica (HAS)", sendo ainda maior após a menopausa, efeito que é diretamente dependente da elevação do $\mathrm{IMC}^{12}$.

$\mathrm{Na}$ obesidade abdominal os adipócitos são metabolicamente mais ativos e os ácidos graxos resultantes de sua lipólise migram para o figado, ocupando os receptores de insulina e resultando em hiperinsulinemia9; esta, em altas concentrações, liga-se aos receptores de IGF-I, elevando a produção de androgênios, que somada com a redução do SHBG, ocasionada pela obesidade, provoca aumento na biodisponibilidade dos estrogênios, principalmente da estrona $^{13}$. No endométrio este incremento do IGF-I representa também um possível fator de proliferação local, podendo acarretar em hiperplasia e câncer do endométrio ${ }^{12}$.

Apesar da associação câncer de endométrio e obesidade estar bem estabelecida, a maioria dos estudos tem avaliado os fatores de risco em mulheres já portadoras do câncer. Entretanto, escassos são os estudos que analisaram se mulheres obesas deveriam ser submetidas a uma avaliação do endométrio, mesmo não apresentando qualquer sintoma, como sangramento uterino anormal. Por isso, o presente estudo teve por objetivo avaliar a prevalência de lesões endometriais, como pólipos, hiperplasia e câncer de endométrio em mulheres obesas assintomáticas, além do caracterizar possíveis fatores de risco associados. 


\section{Métodos}

Em estudo de corte transversal foram avaliadas 94 mulheres obesas, no Ambulatório de Endocrinologia Ginecológica do Departamento de Obstetrícia e Ginecologia da Faculdade de Ciências Médicas da Santa Casa de Misericórdia de São Paulo/Brasil, entre dezembro de 2004 e fevereiro de 2006. O estudo foi aprovado pelo Comitê de Ética da instituição e todas as participantes assinaram um termo de consentimento informado.

Os critérios de inclusão foram mulheres com $\mathrm{IMC}\left(\mathrm{Kg} / \mathrm{m}^{2}\right) \geq 30$ e idade $\geq 18$ anos; e os de exclusão foram queixa de sangramento uterino disfuncional ou após a menopausa, uso de qualquer terapia hormonal nos últimos seis meses e/ou presença de tumores ovarianos. Todas responderam um questionário para obter: idade (anos), cor (branca ou não), tempo de obesidade (anos), hábitos (fumo, álcool), paridade (número de partos de feto viável vivo ou morto), antecedentes pessoais (HAS, diabetes e uso de medicações), tempo de pós-menopausa (anos) e características menstruais.

A determinação das pressões arteriais sistólica (PAS) e diastólica (PAD) foi medida pelo método auscultatório, com esfignomanômetro de coluna de mercúrio, sendo considerados como alteradas PAS $\geq 140 \mathrm{mmHg}$ e a $P A D \geq 90 \mathrm{mmHg}^{14}$.

A avaliação da obesidade foi feita por métodos antropométricos, como peso, altura, e medida da circunferência da cintura abdominal (CA). Pelo IMC caracterizou-se pré-obeso, quando se situava entre 25 e 29,9 (Kg/m²); obesidade grau I entre 30 e 34,9; II entre 35 e 39,9 e III $\geq 40^{15}$. O peso (Kg) foi medido em balança hospitalar e a altura (metros) em estadiômetro de parede, com as mulheres vestindo aventais, descalças e em pé. A $\mathrm{CA}(\mathrm{cm})$ foi medida com fita métrica na linha média entre o rebordo costal e a crista ilíaca ântero-superior, sob expiração e em pé, sendo o valor $>88 \mathrm{~cm}$ caracterizado como obesidade abdominal ${ }^{16}$. A seguir, foram realizados exames físico e ginecológico completos.

Todas as participantes foram submetidas à coleta de sangue, em veia periférica para os seguintes exames bioquímicos: IGF-I (ng/ $\mathrm{mL})$, estrona $(E l ; \mathrm{pg} / \mathrm{mL}), \mathrm{FSH}(\mathrm{mU} / \mathrm{mL})$, colesterol total com frações $(\mathrm{mg} / \mathrm{dL})$, insulina $(\mathrm{uU} / \mathrm{mL})$ e glicemia de jejum $(\mathrm{mg} / \mathrm{dL})$. Os exames foram considerados normais quando os valores estavam abaixo de: colesterol 200, LDL-c 130, HDL-c acima de 4017; glicemia 100 , insulina $23^{18}$.

A avaliação do endométrio foi iniciada pela ultra-sonografia endovaginal (USG) com transdutor vaginal de $6,5 \mathrm{MHz}$ com aparelho Hitachi EUB-405 (Japão), realizando imagens em planos coronal e sagital da cavidade endometrial para medida do eco endometrial $(\mathrm{mm})$, todos realizados por um único examinador. A seguir, foi realizada biópsia com uma cânula de $3,1 \mathrm{~mm}$ (Pipelle de Cornier, Laboratoire C.C.D., Paris-France $)^{19}$ e o material obtido encaminhado para análise histológica. A histeroscopia diagnóstica foi indicada somente nos casos onde foram sugeridas alterações endometriais na USG, como pólipo, mioma submucoso, irregularidade da cavidade endometrial ou espessamento endometrial $\geq$ $5 \mathrm{~mm}$ nas mulheres após a menopausa ${ }^{20}$. Foi realizada também, nos casos de patologia endometrial revelado pelo estudo histológico. Para as mulheres em idade reprodutiva, todos os exames foram realizados na fase folicular do ciclo menstrual.

\begin{tabular}{|c|c|c|c|c|}
\hline \multicolumn{5}{|c|}{ Tabela I - Características clínicas da população em estudo } \\
\hline \multirow[b]{2}{*}{ Variáveis } & \multicolumn{2}{|c|}{ Idade Reprodutiva (47) } & \multicolumn{2}{|c|}{ Após a Menopausa (47) } \\
\hline & $\begin{array}{c}\text { EA(6) } \\
\text { Média } \pm \text { EP }\end{array}$ & $\begin{array}{c}\text { EN(4I) } \\
\text { Média } \pm \text { EP }\end{array}$ & $\begin{array}{c}\text { EA(19) } \\
\text { Média } \pm \text { EP }\end{array}$ & $\begin{array}{c}\text { EN(28) } \\
\text { Média } \pm \text { EP }\end{array}$ \\
\hline $\begin{array}{l}\text { Idade(anos)* } \\
\text { Obesidade (anos) } \\
\text { Menopausa (anos) } \\
\text { Paridade (n }{ }^{\circ} \text { ) } \\
\text { Tabagismo\%(n) } \\
\text { HAS\%(n)** }\end{array}$ & $\begin{array}{c}2,5 \pm 0,8 \\
16,7(1) \\
66,7(4)\end{array}$ & $\begin{array}{c}38,6 \pm 1,3 \\
11,2 \pm 1,4 \\
- \\
1,9 \pm 0,2 \\
34,1(14) \\
17,1(7)\end{array}$ & $\begin{array}{c}59,1 \pm 2,2 \\
16,9 \pm 1,9 \\
10,2 \pm 2,3 \\
2,4 \pm 0,4 \\
15,8(3) \\
68,4(13)\end{array}$ & $\begin{array}{l}55,9 \pm 1,4 \\
19,5 \pm 2,4 \\
7,8 \pm 1,2 \\
3,1 \pm 0,4 \\
35,7(10) \\
57,1(16)\end{array}$ \\
\hline
\end{tabular}

EA: endométrio alterado; EN: endométrio normal; EP: erro padrão. Apenas para 0 grupo em idade reprodutiva: $*<0,003$; $* * * 0,021$.

$\mathrm{Na}$ análise estatística foram utilizados os testes de MannWhitney e t de Student para as variáveis quantitativas, com o cálculo da média e erro padrão (EP), e para as qualitativas o $\chi^{2}$ e Exato de Fisher, caracterizando-se associação estatística significativa quando $p<0,05$. Foi realizada regressão logística multivariada com critério de seleção de variáveis Stepwise, onde a variável dependente foi o endométrio alterado e a independente, as demais variáveis. O risco estimado de desenvolver endométrio alterado foi calculado pelo odds ratio (OR), com intervalo de confiança (IC) de 95\%.

\section{Resultados}

A população estudada foi composta de 94 mulheres obesas assintomáticas, 47 destas em idade reprodutiva e 47 na pósmenopausa. Após a realização dos exames, e baseados nas alterações endometriais confirmadas pela biópsia e/ou histeroscopia, os dois grupos foram subdivididos em endométrio alterado (EA), sendo seis em idade reprodutiva e 19 após a menopausa; e endométrio normal (EN), sendo $4 \mathrm{I}$ em idade reprodutiva e 28 após a menopausa. Portanto, encontramos 12,8\% de alterações endometriais na idade reprodutiva e 40,4\% após a menopausa, apresentando diferença estatística $(p<0,0024)$.

Dentre as características clínicas das mulheres estudadas, $76,6 \%$ das mulheres tanto em idade reprodutiva, quanto após a menopausa, eram brancas, não havendo diferença em relação ao endométrio. Apenas quatro mulheres apresentavam diagnóstico prévio de diabetes, todos associados à HAS, sendo uma em idade reprodutiva e três após a menopausa, todos com endométrio normal.

A Tabela I apresenta as características clínicas da população divididas em quatro grupos. As variáveis que mostraram resultados significativos em relação ao EA foram a média da idade e a porcentagem de mulheres com HAS, mas apenas, nas mulheres em idade reprodutiva.

Em relação às características do exame físico desta população, a altura média das mulheres em idade reprodutiva foi de $1,59 \mathrm{~m}$, e após a menopausa de $1,55 \mathrm{~m}$, não havendo diferença quanto ao endométrio. A Tabela 2 apresenta as características do exame físico da população divididas em quatro grupos. A única variável que teve associação significativa foi a PAS $\geq 140 \mathrm{mmHg}$, tendo o IMC (tanto 


\begin{tabular}{|c|c|c|c|c|}
\hline \multicolumn{5}{|c|}{ Tabela 2 - Características físicas da população em estudo } \\
\hline \multirow[b]{2}{*}{ Variáveis } & \multicolumn{2}{|c|}{ Idade Reprodutiva (47) } & \multicolumn{2}{|c|}{ Após a Menopausa (47) } \\
\hline & $\begin{array}{c}E A(6) \\
\text { Média } \pm E P\end{array}$ & $\begin{array}{c}\text { EN(4I) } \\
\text { Média } \pm \text { EP }\end{array}$ & $\begin{array}{c}E A(19) \\
\text { Média } \pm \text { EP }\end{array}$ & $\begin{array}{c}\text { EN(28) } \\
\text { Média } \pm \text { EP } \\
\end{array}$ \\
\hline $\begin{array}{l}\text { Peso }(\mathrm{Kg}) \\
\text { IMC }\left(\mathrm{Kg} / \mathrm{m}^{2}\right)^{*} \\
\text { CA(cm) } \\
\text { PAS }(\mathrm{mmHg}) \\
\text { PAD }(\mathrm{mmHg}) \\
\text { PAS } \geq 140 \mathrm{mmHg} \%(\mathrm{n})^{* *} \\
\text { PAD } \geq 90 \mathrm{mmHg} \%(\mathrm{n}) \\
\text { IMCI\% } \%(\mathrm{n})^{* * * *} \\
\text { IMCII\% }(\mathrm{n}) * * * * \\
\text { IMCIII\% } \%(\mathrm{n})^{* * * *}\end{array}$ & $\begin{array}{c}100,3 \pm 9,5 \\
39,3 \pm 2,9 \\
118,0 \pm 5,5 \\
128,3 \pm 4,8 \\
86,7 \pm 3,3 \\
* \quad 33,3(2) \\
50,0(3) \\
33,3(2) \\
33,3(2) \\
33,3(2)\end{array}$ & $\begin{array}{c}94,9 \pm 3,1 \\
37,2 \pm 0,9 \\
112,3 \pm 2,2 \\
130,5 \pm 3,0 \\
85,9 \pm 3,1 \\
36,6(15) \\
56,1(23) \\
39,0(16) \\
34,1(14) \\
26,8(11)\end{array}$ & $\begin{array}{l}95,2 \pm 5,0 \\
39,4 \pm 1,7 \\
113,8 \pm 2,8 \\
147,4 \pm 5,2 \\
93,2 \pm 2,8 \\
73,7(14) \\
68,4(13) \\
26,3(5) \\
36,8(7) \\
36,8(7)\end{array}$ & $\begin{array}{l}87,2 \pm 2,8 \\
36,0 \pm 0,8 \\
111,0 \pm 1,8 \\
137,5 \pm 3,4 \\
89,3 \pm 2,6 \\
42,9(12) \\
50,0(14) \\
60,7(17) \\
21,4(6) \\
17,9(5)\end{array}$ \\
\hline
\end{tabular}

EA: endométrio alterado EN: endométrio normal; $E P$ : erro padrão.

Apenas para 0 grupo após a menopausa: * $p<0,0571$; ${ }^{* *} p<0,0370$; ${ }^{*} * *$ * $p<0,0662$.

Tabela 3 - Comparação entre as determinações bioquímicas

\begin{tabular}{|c|c|c|c|c|}
\hline \multirow[b]{2}{*}{ Variáveis } & \multicolumn{2}{|c|}{ Idade Reprodutiva (47) } & \multicolumn{2}{|c|}{ Apósa Menopausa (47) } \\
\hline & $\begin{array}{c}\text { EA(6) } \\
\text { Média } \pm \text { EP } \\
\end{array}$ & $\begin{array}{c}\text { EN(4I) } \\
\text { Média } \pm \text { EP } \\
\end{array}$ & $\begin{array}{c}E A(19) \\
\text { Média } \pm E P \\
\end{array}$ & $\begin{array}{c}\text { EN(28) } \\
\text { Média } \pm \text { EP }\end{array}$ \\
\hline $\begin{array}{l}\text { IGF-I (ng/mL) } \\
\text { EI (pg/mL)* } \\
\text { Glicemia (mg/dL) } \\
\text { Insulina (uU/mL) } \\
\text { Colesterol(mg/dL)** } \\
\text { LDL-c(mg/dL)*** } \\
\text { Colesterol } 2200 \%(\mathrm{n}) \\
\text { LDL-c } \geq 130 \%(\mathrm{n}) * * * 6\end{array}$ & $\begin{array}{c}180,3 \pm 41,3 \\
55,8 \pm 13,1 \\
87,0 \pm 6,7 \\
13,3 \pm 3,4 \\
216,7 \pm 12,7 \\
134,8 \pm 13,1 \\
66,7(4) \\
83,3(5)\end{array}$ & $\begin{array}{c}228,4 \pm 16,6 \\
77,0 \pm 11,8 \\
87,8 \pm 4,7 \\
12,1 \pm 1,7 \\
187,3 \pm 4,8 \\
114,2 \pm 5,0 \\
34,1(14) \\
24,4(10)\end{array}$ & $\begin{array}{c}142,0 \pm 10,5 \\
46,7 \pm 7,1 \\
97,6 \pm 6,3 \\
15,5 \pm 2,4 \\
224,9 \pm 9,8 \\
139,1 \pm 8,8 \\
78,9(15) \\
63,2(12)\end{array}$ & $\begin{array}{c}153,9 \pm 17,4 \\
31,3 \pm 3,1 \\
93,6 \pm 4,2 \\
12,4 \pm 1,0 \\
221,4 \pm 7,1 \mid \\
28,8 \pm 5,4 \\
57,1(16) \\
35,7(10)\end{array}$ \\
\hline
\end{tabular}

EA: endométrio alterado EN: endométrio normal; EP: erro padrão.

No grupo da idade reprodutiva: *** $p<0,0366$; **** $p<0,0504$; ***** $p<0,0094$

No grupo após a menopausa: * $p<0,0135 ;$ ***** $^{*} p<0,0643$.

a média quanto a porcentagem de I, II e III) resultados muito próximos de serem significativamente estatísticos; resultados válidos apenas no grupo após a menopausa.

Dentre as determinações bioquímicas realizadas, o HDL-c e o VLDL-c não apresentaram diferença entre as mulheres em idade reprodutiva e após a menopausa, nem relação com o endométrio alterado. A Tabela 3 mostra os resultados das determinações bioquímicas, divididos em quatro grupos, podendo ser notado que os significativamente alterados foram a média do colesterol total e do LDL-c, além da porcentagem de LDL-c elevado nas mulheres em idade reprodutiva; após a menopausa a única variável significativa foi a média da estrona (EI), sendo que nessas mulheres, a porcentagem de LDL-c elevado teve valor próximo de um resultado significativo.

$\mathrm{Na}$ idade reprodutiva, o eco endometrial mostrou-se alterado em 6,4\% (três mulheres) fazendo diagnóstico de patologia endometrial em $50 \%$ dos casos. A média do eco endometrial mensurada no grupo de endométrio normal foi de $7 \mathrm{~mm}$, e no endométrio alterado de $1 \mathrm{Imm}$, tendo diferença estatística com $p<0,0273$. Na pós-menopausa esteve alterado em $42,5 \%$ (20) dos casos, tendo apenas um falso-positivo, diagnosticando 100\% das patologias endometriais. No endométrio normal, a média mensurada foi de $4 \mathrm{~mm}$ e no alterado de $10 \mathrm{~mm}$, tendo também diferença estatística com $p<0,0001$.

Nos resultados das biópsias de endométrio na idade reprodutiva obtivemos $26(55,3 \%)$ de casos de endométrio proliferativo, sete $(14,9 \%)$ com presença de glândulas endometriais, quatro $(8,6 \%)$ de endométrio secretor, três $(6,4 \%)$ de material insuficiente, três $(6,4 \%)$ casos de estenose de canal cervical, dois (4,2\%) de pólipos, um (2,1\%) de hiperplasia sem atipia e um $(2,1 \%)$ de adenocarcinoma. Na menopausa obtivemos 19 casos (40,4\%) com material insuficiente, dez (21,3\%) com presença de glândulas endometriais, oito (17\%) com estenose de canal cervical, quatro $(8,6 \%)$ casos de endométrio proliferativo, três $(6,4 \%)$ de pólipos, um $(2,1 \%)$ de endométrio atrófico, um (2,1\%) de hiperplasia com atipia e um $(2,1 \%)$ de adenocarcinoma. No total, foram diagnosticados três casos de lesões endometriais de alto risco, sendo dois cânceres de endométrio e uma hiperplasia com atipia, que tinham como características: idade superior a 49 anos, obesidade abdominal com IMC III, eram brancas, não-tabagistas, com paridade baixa (I,6 filhos), HAS, com elevação da PAS, colesterol total e LDL-c.

$\mathrm{Na}$ idade reprodutiva foram realizadas seis histeroscopias diagnósticas, sendo quatro casos de pólipos, um de hiperplasia e um de corpo estranho (fio de dispositivo intra-uterino). Na pósmenopausa foram realizadas 19 histeroscopias, sendo 12 casos de lesões polipóides, quatro de lesões polipóides associadas com miomas submucosos, dois de hiperplasia e um de endométrio proliferativo. Os dois casos de câncer de endométrio, por já terem diagnóstico confirmado pela biópsia, não realizaram este exame.

$\mathrm{Na}$ análise de regressão logística multivariada (OR, odds ratio) constatamos que nas mulheres em idade reprodutiva, ter o LDL$c \geq 130 \mathrm{mg} / \mathrm{dL}$ aumentou em 18,1 vezes o risco de apresentar patologia endometrial comparativamente com as de LDL-c normal (IC 95\% de I,4; $p<0,0272$ ). Para as mulheres após a menopausa, estar com a PAS $\geq 140 \mathrm{mmHg}$ elevou esse risco em 5, I vezes (IC 95\% de 1,3; p < 0,0229) e ter LDL-c elevado aumenta em 4,4 vezes (IC 95\% de I,I; $p<0,0354$ ) o risco de endométrio alterado. Mulheres na pós-menopausa exibiram risco 7,8 vezes maior de apresentar endométrio alterado, comparativamente as que estavam na idade reprodutiva (IC 95\% de 2,0; p < 0,0029). Ter o LDL-c elevado, independente da faixa etária, elevou esse risco em 4,7 vezes (IC 95\% de 1,6; $p<0,0044$ ).

\section{Discussão}

A obesidade é um importante fator de risco para o câncer endometrial, em decorrência da conversão de testosterona em estrona no tecido adiposo, levando a um maior estímulo endometrial. Entretanto, nosso objetivo foi avaliar o endométrio de mulheres obesas assintomáticas e caracterizar o quanto a obesidade e outros fatores de risco, podem redundar em um maior risco de lesões precursoras ou câncer de endométrio.

O presente estudo incluiu mulheres obesas tanto na idade reprodutiva, quanto após a menopausa, pois ambas exibem perfil 
de risco para o câncer endometrial; as obesas em idade reprodutiva, pela maior prevalência de anovulação ${ }^{13}$ e as na pós-menopausa, pela maior biodisponibilidade dos estrogênios, resultantes do aumento da conversão periférica dos androgênios em estrona, da diminuição da $\mathrm{SHBG}^{9}$ e da hiperinsulinemia crônica, comum nestas mulheres".

A obesidade foi caracterizada pelo IMC $\left(\mathrm{Kg} / \mathrm{m}^{2}\right)$, por se tratar de método rápido e de baixo custo ${ }^{15}$ para estudos populacionais ${ }^{7}$, embora não diferencie da obesidade abdominal, que também predispõe a um maior risco de câncer de endométrio ${ }^{21}$; para contornar isso, utilizamos também a medida da CA, que mostrou obesidade abdominal em 98,9\% das mulheres da nossa casuística.

O endométrio foi avaliado ecograficamente, sempre pelo mesmo examinador, com o objetivo de reduzir o risco de falhas no exame ${ }^{20}$; o eco endometrial mostrou-se alterado $6,4 \%$ na idade reprodutiva tendo três casos de falso-negativos, sendo um caso de câncer de endométrio com eco de $12 \mathrm{~mm}$, fazendo diagnóstico em apenas $50 \%$ dos casos. Na pós-menopausa esteve alterado em $42,5 \%$, tendo apenas um falso-positivo, diagnosticando $100 \%$ das patologias endometriais nesta faixa etária. O diagnóstico de patologias endometriais feito apenas pela USG foi de $88 \%$ no total, coincidindo com os achados da literatura, em torno de $80 \%$ 22 , que obrigou a complementação do estudo endometrial.

A complementação do estudo foi feita pela biópsia endometrial, que pode diagnosticar até $90 \%$ dos casos de câncer de endométrio em mulheres na idade reprodutiva e $99 \%$ após a menopausa, representando expressivo benefício, em termos de segurança, custo e aceitação pelas mulheres. $O$ acesso ao endométrio ocorre em torno de $65 \%$ dos casos, sendo que os casos de amostras inadequadas, em geral, podem-se atribuir à atrofia endometrial, principalmente após a menopausa' ${ }^{19}$; no nosso estudo obtivemos material endometrial em $64,9 \%$, diagnosticando dois casos de adenocarcinoma $(2,2 \%)$ e um de hiperplasia atípica $(1,1 \%)$. Na idade reprodutiva este diagnóstico ocorreu em $87,2 \%$ e após a menopausa em 42,5\%. Nossos resultados de cânceres foram superiores aos observados na literatura, em diversas populações, oscilando entre $0,07 \%$ a $1,9 \%$, porém, não em obesas exclusivas, o que talvez justifique esta nossa diferença.

A histeroscopia diagnóstica foi realizada para confirmar patologia endometrial, sendo considerada o padrão ouro, com sensibilidade de $90 \%$ nas lesões atróficas, 95\% nos pólipos, $87 \%$ nas hiperplasias atípicas, 92\% nas típicas e 94\% nos carcinomas de endométrio ${ }^{22}$. Na idade reprodutiva encontramos $12,8 \%$ de alterações endometriais confirmadas pela biópsia e/ou histeroscopia e 40,4\% após a menopausa. Reslová et al. (1999) avaliando mulheres que apresentavam sangramento uterino disfuncional, como fator de risco, constataram alterações endometriais em 15\% na idade reprodutiva e em 26\% após a menopausa ${ }^{23}$. Portanto, no nosso estudo, obtivemos um número maior de patologia endometrial em mulheres após a menopausa, mesmo assintomáticas, devido provavelmente, ao fato da obesidade se associar mais com lesões precursoras do que o sangramento nesta faixa etária.

Os pólipos endometriais são considerados lesões precursoras, apesar de raramente sofrerem transformação maligna (0,5-4,8\%); apresentam risco de hiperplasia típica em torno de $25 \%$ e atípica ao redor de 3, $1 \% 23$. Após a menopausa, a associação entre obesidade,
HAS e pólipos pode elevar o risco de câncer endometrial ${ }^{\mid 1-12}$, sendo portanto lesões preocupantes para a população estudada.

$\mathrm{Na}$ obesidade, já existe uma intensa associação com a HAS e alterações do colesterol, principalmente após a menopausa, onde obtivemos mais de $60 \%$ das mulheres com estas patologias, sendo em torno de 30\% na idade reprodutiva; quando associadas à patologia endometrial, apresentaram porcentagens ainda mais elevadas.

Nas mulheres com endométrio alterado, nas duas faixas etárias, - IMC esteve mais elevado do que naquelas com endométrio normal, confirmando o estudo de Avis \& Crawford (200I), que relacionou o aumento do IMC com patologia endometrial ${ }^{24}$. As demais variáveis, idade, peso, CA, insulina estavam elevadas no grupo do endométrio alterado nas duas faixas etárias; assim como o tempo de menopausa e o de obesidade (este apenas na idade reprodutiva). Não observamos associação entre patologia endometrial e elevação da IGF-I, desencadeada pela obesidade e insulinemia ${ }^{25}$. O tabagismo foi mais freqüente nos grupos de endométrio normal, parecendo se apresentar como fator de proteção para o câncer endometrial|'

As mulheres após a menopausa absorveram um total de $76 \%$ das patologias endometriais encontradas, apresentando associação significativa com aumento da estrona, e mais intensamente com as elevações da PAS e LDL-c. A obesidade associada ao status menopausal, elevou o risco de lesão endometrial, sendo estas mulheres, candidatas à biópsia do endométrio.

$\mathrm{Na}$ idade reprodutiva, tivemos um número pequeno de casos de lesões endometriais, que para serem conclusivos, necessitariam de mais estudos, mas pudemos verificar a associação com elevação da idade, HAS, colesterol e principalmente LDL-C; poderíamos apenas sugerir uma maior atenção àquelas com idade superior a 40 anos (a idade mínima de patologia endometrial encontrada foi de 42 anos), que apresentassem essas associações, que foram muito semelhantes às mulheres na pós-menopausa.

Nas mulheres obesas, principalmente com IMC III, o exame ginecológico é, às vezes, dificultado pela obesidade vaginal, sendo prejudicada a visualização do colo uterino, que em alguns casos, foi realizada com auxílio da pinça de Pozzi. Este é apenas um dos problemas que as mulheres obesas enfrentam durante uma consulta ginecológica, além do preconceito dos próprios médicos, da vergonha de expor seu corpo e dificuldade de se posicionar na mesa ginecológica. Essas mulheres, conscientes das dificuldades referidas e ainda portadoras de fatores de risco para patologia endometrial acabam freqüentando muito pouco os consultórios ginecológicos, retardando, dessa forma, o diagnóstico precoce das alterações endometriais.

\section{ConclusÃo}

O aumento de incidência da obesidade nos últimos anos tem propiciado maior risco para o câncer de endométrio.

A prevalência de lesões endometriais em mulheres obesas assintomáticas foi maior nas mulheres após a menopausa, principalmente quando associada com HAS e elevação do LDL-c. Essas mulheres deveriam ser submetidas a exames rotineiros de ultrasonografia endovaginal associada à biópsia endometrial, visando o 
Gouvela DAC et AL.

diagnóstico precoce do câncer de endométrio.

Assim, a triagem de mulheres assintomáticas, mas com conhecidos fatores de risco, poderá contribuir para o diagnóstico precoce do câncer de endométrio, condição imprescindível para o prognóstico da doente.

\section{Conflito de interesse: não há.}

\section{SUMMARY}

\section{Prevalence of endometrial injury in asymptomatic obese WOMEN}

OBIECTIVE. Obesity represents the most important risk factor for endometrial pathology. This study aimed to evaluate the prevalence of endometrial injuries, such as polyps, hyperplasia and endometrial cancer in asymptomatic obese women, as well as to recognize the associated risk factors .

Methods. A cross-sectional study was conducted between December 2004 and February 2006. Ninety-four obese (body mass index $\geq 30 \mathrm{~kg} / \mathrm{m}^{2}$ ) women were evaluated, divided in two groups of 47 participants each: pre-menopausal and postmenopausal women. Clinical characteristics, physical exams (anthropometric and gynecological), blood count and endometrial assessment by vaginal ultrasonography, biopsy, and hysteroscopy (only for confirmed endometrial pathology), were the variables appraised.

RESULTS. In pre-menopausal women, $12.8 \%$ of cases had endometrial pathology statistically associated to age, hypertension, hypercholesterolemia and LDL-c increase. In the post-menopausal women, $40.4 \%$ of cases had a pathology identified as statistically associated with hypertension, LDL-c and estrone increase. Two cases of endometrial cancer were identified, one in each group.

Conclusions. The increased incidence of obesity over recent years has increased risk factors of endometrial cancer. In premenopausal women only a small number of cases with endometrial alterations was observed. Therefore, it is suggested that greater attention be given to those over 40 years of age, associated with hypertension and/or higher LDL-c. The menopausal status increases risk of endometrial injury, and when associated with hypertension, $L D L-c$ and/or estrone increase, women become candidates to biopsy aiming for an early diagnosis of cancer, a decisive factor for a favorable prognosis. [Rev Assoc Med Bras 2007; 53(4): 344-8]

KEY WORDS: Obesity. Endometrium. Pre-menopausal. Postmenopausal. Hypertension. Cholesterol.

\section{REFERÊNCIAS}

I. Coutinho W. Latin América consensus on obesity. Arq Bras Endocrinol Metab. 1999:43:21-67.

2. Binfa EL, Blumel MJH. Obesidad, estrógenos y salud de la mujer. Rev Chil Obstet Ginecol. 2001;66:340-6.

3. Blümel JE, Castelo Branco C, Rocangliolo ME, Binfa L, Tacla X, Mamani L. Changes in body mass index around menopause: a population study of Chilean woman. Menopause. 2001;8:239-44.

4.WHO. World Health Organization. Global strategy on diet, physical activity and health. [cited 2006 oct 6]. Avaliable from: http:// www.who.int/dietphysicalactivity/publications/facts/obesity/.
5. Mondini L, Monteiro CA. The stage of nutrition transition in different Brasilian regions. Rev Arch Latinoam Nutr. 1997;47(1): 17-21.

6.IBGE. Instituto Brasileiro de Geografia e Estatística. Estado nutricional, precisão das estimativas e totais da população 2002-2003 [citado 6 Out 2006]. Disponível em; http://www.ibge.gov.br/home/estatistica/ populacao/condicaodevida/pof/2002analise/defaulttab.shtm.

7. Anderson JW, Konz EC. Obesity and disease management: effects of weight loss on comorbid conditions. The North American Association for the Study of Obesity. Obes Res. 2001;9:326-34.

8. Soliman PT, Oh JC, Schmeler KM, Sun CC, Slomovitz BM, Gershenson DM, et al. Risk factors for young premenopausal women with endometrial cancer. Obstet Gynecol. 2005; 105:575-80.

9. Kaaks R, Lukanova A, Kurzer MS. Obesity, endogenous hormones, and endometrial cancer risk: a synthetic review. Cancer Epidemiol Biomarkers Prev. 2002; 11:1531-43.

I0. Göl K, Saraçoglu F, Ekici A, Sahin I. Endometrial patterns and endocrinologic characteristics of asymptomatic menopausal women. Gynecol Endocrinol. 2001;15:63-7.

II. Weiderpass E, Persson I, Adami HO, Magnusson C, Lindgren A, Baron JA. Body size in different periods of life, diabetes mellitus, hypertension, and risk postmenopausal endometrial cancer (Swenden). Cancer Causes Control. 2000; 1 I:185-92.

12. Serin IS, Ozçelik B, Basbug M, Ozsahin O, Yilmazsoy A, Erez R. Effects of hypertension and obesity on endometrial thickness. Eur J Obstet Gynecol Reprod Biol. 2003;109:72-5.

13. Webb PM. Commentary: weight gain, weight loss, and endometrial cancer. Int J Epidemiol. 2006;35:166-8.

14. Sociedade Brasileira de Hipertensão. Portal da hipertensão. IV Diretrizes Brasileiras de Hipertensão, 2005 [citado | Out 2006]. Disponível em: http://www.sbh.org.br.

15. National Cholesterol Education Program (NCEP) Expert panel on detection, evaluation and treatment of high blood cholesterol in adults. Executive Summary of a third report. JAMA. 2001;285:2486-97.

16. Garaulet M, Pérez-Llamas F, Zamora S, Tebar FJ. Estúdio comparativo del tipo de obesidad pré y posmenopáusicas: relación con tamaño adipocitário, la composición de la grasa y diferentes variables endocrinas, metabólicas, nutricionales y psicológicas. Med Clin (Barcelona). 2003; 10:784-802.

17. III Diretrizes Brasileiras de dislipidemia e prevenção de aterosclerose. Arq Bras Cardiol. 2005:84(supll).

18. Sociedade Brasileira de Diabetes. Valores de glicemia para diagnóstico de diabetes. [citado 02 jan 2006]. Disponível em: http:// www.diabetes.org.br/diabetes/exames/valoresdeglicemia.php.

19. Bahamondes L. Câncer de endométrio: o que considerar? Rev Assoc Med Bras. 2004;50(I).

20. Gerber J, Sozanski L, Suchocki S. The risk factors of endometrial cancer. Ginekol Pol. 2001:72(I2A):|4|8-22.

2I. Soares Jr JM, Espósito IC, Patriarca MT, Baracat EC. Obesidade. In: Baracat EC, Rodrigues de Lima G, editores. Guias de medicina ambulatorial e hospitalar UNIFESP/Escola Paulista de Medicina. São Paulo: Manole; 2005 p.385-94.

22. Escobar DP, Alvarado CLS, Bueno FN. Comparativo entre ultrassonografia transvaginal e histeroscopia diagnóstica em patologia endouterina. Chil Obstet Ginecol. 2000;65:183-7.

23. Reslová T, Tosner J, Resl M, Kugler R, Vávrová I. Endometrial polyps. A clinical study of 245 cases. Arch Gynecol Obstet. 1999;262:133-9.

24. Avis NE, Crawford SL. Menopause and weight (review). Menopause. 2001:8:230-32.

25. Ginzbarg D, Teixeira RJ, Dimetz T, Henriques JLM, Oliveira HC. Terapia de reposição hormonal contínua na pós-menopausa: ênfase no hormônio do crescimento, insulina, fator de crescimento semelhante à insulina I (IGF-I) e Proteína Ligadora 3 do IGF (IGFBP-3). Arq Bras Endocrinol Metab. 200।;45. 